\title{
Phase Difference Observations at Spaced Aerials and Their Application to Direction Finding ${ }^{1,2}$
}

\author{
W. C. Bain
}

(August 22, 1960; revised December 15, 1960)

\begin{abstract}
The measurement of phase differences at two aerial pairs, each spaced by three to four wavelengths, was used to give mean bearings over periods of three to five minutes on certain transmitters with frequencies near $6 \mathrm{Mc} / \mathrm{s}$. The results showed that site errors had probably been reduced by this system to less than $0.5 \mathrm{deg}$, and possibly to $0.2 \mathrm{deg}$, which is a value derived on theoretical grounds. The variance of the mean bearings due to lateral deviation was found to be slightly greater than anticipated. However, the results were such as to indicate that the performance of a wide-aperture direction finder should not fall seriously below that which would be expected of it theoretically on the basis of existing knowledge.
\end{abstract}

\section{Introduction}

In the winter of $1954-55$ a number of observations were carried out at the Radio Research Station in which phase differences were measured between the signals picked up at aerials spaced by several wavelengths. Recently it was noted that these results were relevant to the problem of determining the accuracy obtainable from a wide-aperture direction finder, an instrument capable of improved performance in conditions of multimode propagation [Bain, 1956]. It therefore seemed desirable to present this account of the work and its implications here.

The four aerials used were short unipoles; they were placed on the circumference of a circle of diameter $180 \mathrm{~m}$ so that one pair of aerials was alined in the north-south direction and another in the eastwest direction. Inside a hut at the center of the circle each of these pairs of aerials was connected to phasemeasuring equipment of the type described by Ross, Bramley, and Ashwell [1951]. When the receivers were tuned to the desired station, the overall phase balance of the system was checked, and the phase differences between the signals picked up on the north-south and east-west pairs were recorded photographically at three-second intervals for a period not exceeding five minutes, thus giving about 70 values for each. Ionospheric conditions were characteristic of the time in question and were never greatly disturbed. Observations were confined to the hours of daylight.

It is important to note that such a system is not directly suitable for use as an operational direction finder. Not only does it have bearing ambiguities, but the bearing calculated from the phase differences is not in general correct when two rays are incident on it from the same azimuth.

\footnotetext{
1 Contribution from Department of Scientific and Industrial Research, Radio Research Station, Ditton Park, Slough, Bucks., England.

2 Paper presented at the Conference on Transmission Problems Related to High-Frequency Direction Finding, at UCLA, June 21-24, 1960.
}

\section{Results}

\subsection{Method of Analysis}

In reducing the observations a mean $\bar{\psi}$ of each distribution was calculated in the following way. If there are $n$ phase differences $\psi_{k}$ in each distribution, then

$$
\bar{\psi}=\arg \left\{\sum_{k=1}^{n} \exp \left(j \psi_{k}\right)\right\} .
$$

This avoids the ambiguities which can arise if an ordinary arithmetic mean is used.

The standard deviation $(\sigma)$ of the distribution was taken to be the root-mean-square departure from $\bar{\psi}$. For a completely uniform distribution of phase differences the standard deviation would have the value $104^{\circ}$ approximately. A finite number of random phase differences will, of course, give a standard deviation somewhat less than this, probably about $94^{\circ}$ for the numbers of observations used here.

\subsection{Observed Values}

The observations were concentrated upon a few stations which had been found from a previous experiment to give widely spread phase difference distributions. Of these, the most frequently used were the following. The distances and bearings shown are from the site of the equipment at Winkfield, near Windsor (Berkshire).

It will be seen that with the aerial spacing used here, the diameter of the system will be in the range of three to four wavelengths for reception of these stations.

It was indeed found that very large standard deviations were occasionally obtained; a few were so great that they could have arisen from a random distribution of phase differences. A camplete set of 
the standard deviations obtained on these three stations is shown in figure 1 . These can be seen to be well spread out over the range of possible values. However, the north-south and east-west standard deviations are correlated to some extent, for if one is large the other also terds to be large. In the case of Arganda, the north-south phase differences were much more widely spread than the east-west; the mean standard deviations were in the ratio of 2.0 to 1. This probably happened because the Arganda bearing is only $10^{\circ}$ away from the north-south direction, combined with the fact that the incident radiation was spread much more in elevation than in bearing.

\section{Bearings Deduced From Phase Measure- ments}

If the phase difference between north and south aerials is $\phi_{1}$, and that between east and west aerials is $\phi_{2}$, then the bearing $\gamma$ can be taken to be derived from the formula

$$
\tan \gamma=\phi_{2} / \phi_{1}
$$

With widely spaced aerials the values of $\phi_{1}$ and $\phi_{2}$ obtained from the measurements may differ from the true values by some integral multiple of $2 \pi$. However, as the position of the transmitter was known in these experiments the ambiguities could be eliminated by choosing the multiples of $2 \pi$ so as to bring the bearing as nearly as possible to the true bearing of the transmitter. The multiple of $2 \pi$ which had to be used never exceeded 3 in any of the present instances.

Mean bearings were calculated by using the mean phase differences $\bar{\psi}$ from each of the groups of observations; the results obtained are summarized in table 2 , which gives results for the mean of all mean bearings for each station.

It can be seen that the results are consistent with the hypothesis that there is no systematic error in

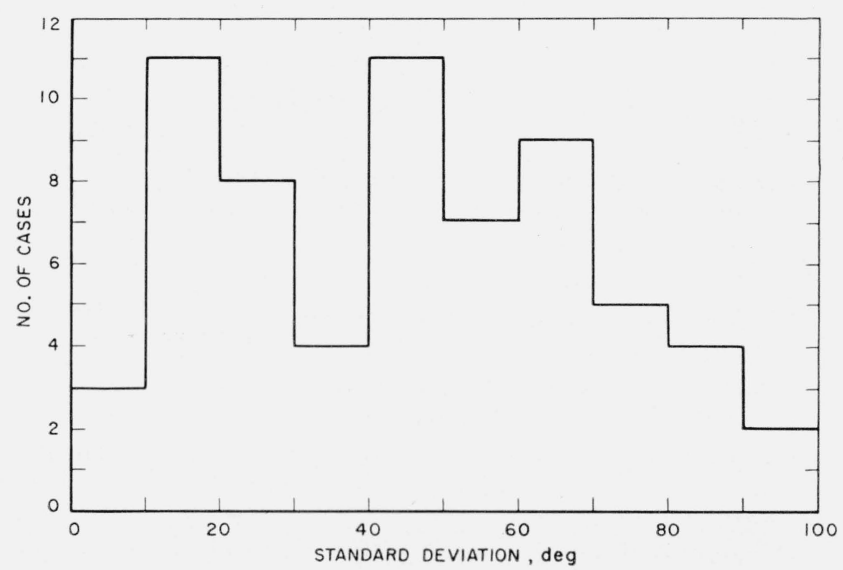

Figure 1. Histogram of the number of cases of standard deviation of the phase difference distributions with values as shown. the observations. However, it is useful to consider in some detail the possible errors which might arise. They can be divided into three main categories for the present purpose:

1. Rapidly fluctuating errors, with periods of the order of seconds; they may be due to wave interference or polarization errors.

2. Lateral deviation errors, with periods of the order of tens of minutes, due to the effect of ionospheric tilts.

3. Site errors, which will be considered here to be virtually constant with time.

The îrst two of these errors would be expected to give rise to a spread of values in the mean bearings obtained for each transmitting station, but the third would lead to a systematic error. On the assumption that the site error due to a narrowaperture Adcock direction finder is $2.5^{\circ}$, it can be estimated from the curves for site-error reduction given by Hopkins and Horner [1949] that the site error for each of the stations in this wide-aperture direction finder would be of the order of $0.2^{\circ}$. Now the range in the fiducial limits for the mean as given in table 2 is sufficiently great to conceal altogether an effect of this magnitude. It is still, of course, possible that the site error of the system is indeed as small as this, but the most that can be said from the experimental evidence here is that it is probably less than $0.5^{\circ}$. This is certainly a worthwhile improvement on a narrow-aperture direction finder. It should be borne in mind that this result was achieved despite the large fluctuations in phase difference commonly found with the transmitters listed in table 1.

The magnitude of the spread of the mean bearings can also be examined to see if it is in accord with what would be expected. To do this some estimate must be obtained of the size of the errors due to rapid fluctuations and to lateral deviation. The error variance $\left(\sigma_{M}^{2}\right)$ due to rapid fluctuations can be assessed from a knowledge of the variance of the north-south and east-west phase differences $\left(\sigma_{1}^{2}\right.$ and

TABLe 1. Details of stations observed

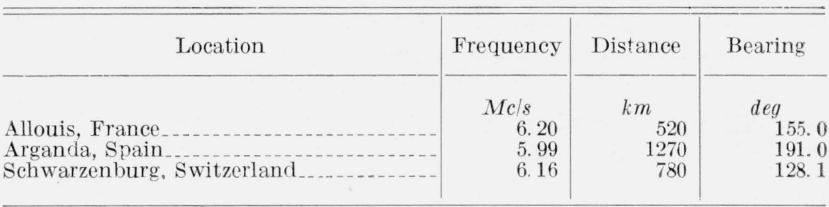

TABLE 2. Errors in the consolidated mean bearing

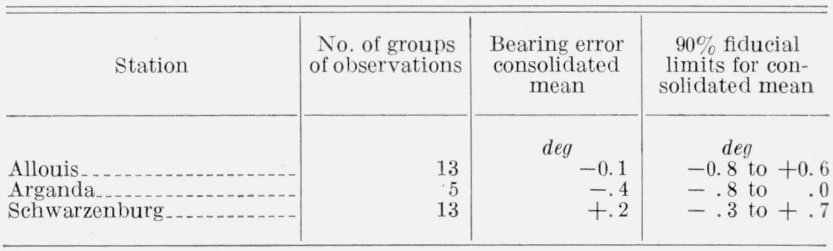


$\sigma_{2}^{2}$, say) in each group. If there are $n_{1}$ north-south phase differences and $n_{2}$ east-west phase differences, and $\theta$ is the bearing of the transmitter, then a reasonable estimate of $\sigma_{M}^{2}$ is given, as is shown in the appendix, by

$$
\sigma_{M}^{2}=\left(\frac{\cos \theta}{2 \beta}\right)^{2} \frac{\sigma_{2}^{2}}{n_{2}}+\left(\frac{\sin \theta}{2 \beta}\right)^{2} \frac{\sigma_{1}^{2}}{n_{1}}
$$

where

$\beta=\frac{\pi d}{\lambda} \cos \delta$,

$d=$ the diameter of the system,

$\lambda=$ the wavelength, and

$\delta=$ the angle of elevation of the incident wave.

Data about the magnitude of ionospheric tilts in the $F$ region can be used to give the variance due to lateral deviation. The figure of $1.2^{\circ}$ determined by Bramley [1953] has been adopted here as the standard deviation of the magnitude of ionospheric tilts in one plane. The expected lateral deviation variance for each station was found from this, again with the assumption that the reflection was from the $F 2$ layer.

In table 3 the variances of the individual mean bearings as obtained in two different ways are compared; if the estimates made are correct the figures in the last two columns should not be significantly different.

The number of observations on Arganda is too small to give much hope of establishing a significant difference between variances, and in fact the values shown in the last two columns for this station are not significantly different. However, for both Allouis and Schwarzenburg it looks as if the estimated variance may be too small, for in each case the difference between observed and estimated variances is nearly significant at the 95 percent level. As the rapidfluctuation variance has been estimated from internal evidence, it looks as if this difference is associated with the lateral-deviation component. However, for present purposes it is sufficient to note that the observed values are not very far from the estimated ones, and there is nothing about these results to suggest that our ideas are seriously wrong about how to apply known information about the ionosphere and direction finding to predict the performance of a wide-aperture direction finder.

TABLE 3. Estimated and observed variances

\begin{tabular}{l|r|r|r|r}
\hline \hline Transmitter & $\begin{array}{c}\text { Estimated } \\
\text { variance due } \\
\text { to ranid fluc- } \\
\text { tuations, } \sigma_{M}^{2}\end{array}$ & $\begin{array}{c}\text { Estimated } \\
\text { variance due } \\
\text { to lateral } \\
\text { deviation }\end{array}$ & $\begin{array}{c}\text { Estimated } \\
\text { variance of } \\
\text { individual } \\
\text { mean bear- } \\
\text { ings (sum of } \\
\text { previous 2 } \\
\text { columns) }\end{array}$ & $\begin{array}{c}\text { Observed } \\
\text { variance of } \\
\text { individual } \\
\text { mean bear- } \\
\text { ings }\end{array}$ \\
\hline & $\operatorname{deg}^{2}$ & $\operatorname{deg}^{2}$ & $\operatorname{deg}^{2}$ & deg $^{2}$ \\
Allouis_... & 1.17 & 1.25 & 2.05 \\
Arganda & 0.08 & 0.32 & 0.42 & 0.14 \\
Schwarzenburg & .10 & .54 & .61 & 1.08
\end{tabular}

\section{Conclusions}

The mean bearing indicated by the phase-difference measuring system considered here, which is of three to four wavelengths in aperture, is likely to be accurate to $\pm 0.5^{\circ}$, and may be as good as $0.2^{\circ}$. To achieve this accuracy time-averaging over a few minutes had to be applied, but in an operational wideaperture direction finder the fast bearing fluctuations should be smaller than was the case here and should require less time averaging to reduce them to an acceptable level. In the practical direction finder the time required to reduce the variance due to lateral deviation of the rays will probably be the same as here, and several hours would be needed to average out its effects. In the present experiments the variance ascribed to lateral deviation turned out to be somewhat larger than expected, but the difference was not so great as to suggest that large unanticipated effects were present. There are, therefore, grounds for hope that wide-aperture direction finders will be capable of the performance expected of them on the basis of existing knowledge.

This work was carried out as part of the program of the Radio Research Board of the Department of Scientific and Industrial Research, and this note is published by permission of the Director of Radio Research.

\section{References}

Bain, W. C., The theoretical design of direction-finding systems for high frequencies, Proc. I.E.E. 103, pt. B, p. 113 (1956).

Bramley, E. N., Direction-finding studies of large-scale ionospheric irregularities, Proc. Roy. Soc. A220, p. 39 (1953)

Hopkins, H. G., and F. Horner, Direction-finding site errors at very high frequencies, Proc. I.E.E. 96, pt. III, p. 321 (1949)

Ross, W., E. N. Bramley, and G. E. Ashwell, A phase-comparison method of measuring the direction of arrival of ionospheric radio waves, Proc. I.E.E. 98, pt. III, p. 294 (1951).

\section{Appendix. Error Variance in the Mean Bearing of a Group of Observations Due to Rapid Fluctuations in the Phase Readings}

The true bearing here will be taken to be given by the expression

$$
\gamma=\arctan \left(\phi_{2} / \phi_{1}\right)
$$

where $\phi_{1}=2 \beta \cos \gamma, \phi_{2}=2 \beta \sin \gamma$.

The error in bearing $\delta \gamma$ is then given by

$$
\delta \gamma=\frac{\cos \gamma \delta \phi_{2}-\sin \gamma \delta \phi_{1}}{2 \beta}
$$


The north-south phase difference, which is here $\left(\phi_{1}+\delta \phi_{1}\right)$, has a variance $\sigma_{1}^{2}$ given by

$$
\sigma_{1}^{2}=\overline{\left(\delta \phi_{1}\right)^{2}}
$$

using the bar to denote an average. If there are $n_{1}$ observations of this phase difference, the variance of the mean is given by $\overline{\left(\delta \phi_{1}\right)^{2}} / n_{1}$. Similarly, the variance of the mean of the east-west phase distribution is $\overline{\left(\delta \phi_{2}\right)^{2}} / n_{2}$. The mean value of the bearing error is given by

$$
\overline{\delta \gamma}=\frac{\cos \gamma \overline{\delta \phi_{2}}-\sin \gamma \overline{\delta \phi_{1}}}{2 \beta}
$$

Hence the variance of the mean bearing is given by

$$
\sigma_{M}^{2}=\left(\frac{\cos \gamma}{2 \beta}\right)^{2} \frac{\sigma_{2}^{2}}{n_{2}}+\left(\frac{\sin \gamma}{2 \beta}\right)^{2} \frac{\sigma_{1}^{2}}{n_{1}}
$$

This derivation applies only when $\delta \phi_{1}$ and $\delta \phi_{2}$ are uncorrelated. However the formula has been checked by calculating bearings for each individual pair of phase differences for certain groups; the standard deviation obtained has been in each case quite close to the value given by the above formula. 